\title{
Cytoskeleton alterations in melanoma: aberrant expression of cortactin, an actin-binding adapter protein, correlates with melanocytic tumor progression
}

\author{
Xu-Zhi Xu' ${ }^{1}$, Marileila Varella Garcia ${ }^{2}$, Tian-yu Li ${ }^{3}$, Li-Yan Khor ${ }^{4}$, R Sujatha Gajapathy ${ }^{2}$, \\ Cindy Spittle ${ }^{5}$, Scott Weed ${ }^{6}$, Stuart R Lessin' ${ }^{7}$ and Hong $\mathrm{Wu}^{1}$ \\ ${ }^{1}$ Department of Pathology, Fox Chase Cancer Center, Philadelphia, PA, USA; ${ }^{2}$ Department of Medicine/ \\ Medical Oncology, University of Colorado Health Sciences Center, Denver, CO, USA; ${ }^{3}$ Facility of Biostatistics, \\ Fox Chase Cancer Center, Philadelphia, PA, USA; ${ }^{4}$ Department of Radiation Oncology, Fox Chase Cancer \\ Center, Philadelphia, PA, USA; ${ }^{5}$ Facility of Biomarkers, Fox Chase Cancer Center, Philadelphia, PA, USA; \\ ${ }^{6}$ Department of Neurobiology and Anatomy, Program in Cancer Cell Biology, Mary Babb Randolph Cancer \\ Center, West Virginia University and ${ }^{7}$ Department of Medicine, Fox Chase Cancer Center, Philadelphia,
}

$P A, U S A$

\begin{abstract}
Cortactin is a multidomain actin-binding protein important for the functions of cytoskeleton by regulating cortical actin dynamics. It is involved in a diverse array of basic cellular functions. Tumorigenesis and tumor progression involves alterations in actin cytoskeleton proteins. We sought to study the role of cortactin in melanocytic tumor progression using immunohistochemistry on human tissues. The results reveal quantitative differences between benign and malignant lesions. Significantly higher cortactin expression is found in melanomas than in nevi $(P<0.0001)$, with levels greater in metastatic than in invasive melanomas $(P<0.05)$. Qualitatively, tumor tissues often show aberrant cortactin localization at the cell periphery, corresponding to its colocalization with filamentous actin in cell cortex of cultured melanoma cells. This suggests an additional level of protein dysregulation. Furthermore, in patients with metastatic disease, high-level cortactin expression correlates with poor disease-specific survival. Our data, in conjunction with outcome data on several other types of human cancers and experimental data from melanoma cell lines, supports a potential role of aberrant cortactin expression in melanoma tumor progression and a rational for targeting key elements of actinsignaling pathway for developmental therapeutics in melanomas.

Modern Pathology (2010) 23, 187-196; doi:10.1038/modpathol.2009.157; published online 6 November 2009
\end{abstract}

Keywords: melanoma; cortactin; metastasis; actin cytoskeleton

\section{Introduction}

Deaths from cutaneous melanoma usually result from metastatic dissemination of invasive tumors. Both invasive melanomas and benign compound or dermal nevi are proliferations of melanocytic cells

Correspondence: Dr H Wu, Department of Pathology, Fox Chase Cancer Center, 333 Cottman Avenue, Philadelphia, PA 19111, USA.

E-mail: hong.wu@fccc.edu

Received 16 March 2009; revised 19 July 2009; accepted 20 July 2009; published online 6 November 2009 in the dermis. In contrast to nevus cells, melanoma cells show cytologic atypia, an escape from cell cycle arrest and aberrant interaction with the dermal environment manifested by a lack of progressive maturation, expansile cellular growth and a variable host immune response. ${ }^{1,2}$ Tumorigenic invasive tumor cells acquire the capacity to migrate, breach into lymphovascular circulation and survive in remote body sites. Active remodeling of a cell's actin cytoskeleton is essential for tumor cell invasion and metastasis. ${ }^{3}$ Altered expression of components of the actin cytoskeleton can also contribute to cellular transformation and tumorigenesis. ${ }^{4}$ 
In additional to a plethora of experimental data, studies using human tissues have also demonstrated over-expression of proteins involved in actin cytoskeleton regulation in colorectal, lung, breast, head and neck and liver carcinomas (reviewed in ref. 3).

Cortactin is a multidomain actin-binding and adaptor/scaffolding protein that is involved in a wide range of cellular functions, including endocytosis, cell migration, invasion, adhesion and neural synaptic organization (reviewed in ${ }^{5-8}$ ). The N-terminus of the protein contains domains that bind to filamentous (F-)actin and Arp2/3 complex, a key machinery in formations of branched actin network that is required for breaking down of pre-existing and establishment of new membrane-cytoskeleton structures. ${ }^{9}$ The C-terminus contains a proline-rich region with sites of serine, threonine and tyrosine phosphorylation, and an SH3 domain that interacts with a variety of cellular proteins, including regulators of actin nucleation (N-WASP and WIP), endocytosis (dynamin, Hip1R and CD2AP) and scaffolding (Shanks). The gene encoding human cortactin $(C T T N)$ is a key component of the $11 \mathrm{q} 13$ amplicon, a chromosomal region frequently amplified in a subset of human breast, head and neck, hepatocellular and bladder carcinomas. Clinical correlative studies have identified CTTN gene amplification and corresponding protein overexpression to be associated with poor clinical outcome, presumably by the enhancement of tumor cell movement and metastasis. ${ }^{10-12}$

A study using a human melanoma cell line A375 shows that cortactin is required for the formation of invadopodia. ${ }^{13}$ In murine melanoma cell lines, cortactin is the only phosphoprotein that demonstrates overexpression, hyperphosphorylation in tyrosine as well as colocalization with tyrosine kinase Fyn in cells with high metastatic potential. ${ }^{14}$ Characteristics of the protein identified in other cell types also make it a particularly promising molecule to study in melanoma tumorigenesis. The aim of this study is to evaluate cortactin expression and gene amplification in human melanocytic lesions.

\section{Materials and methods}

\section{Cell Lines and Cell Culture}

Human melanoma cell lines (1205Lu, WM9 and WM1158) kindly provided by Dr M Herlyn (Wistar Institute, Philadelphia, PA) were grown in ' $2 \%$ tumor' media with $2 \%$ fetal bovine serum (FBS) and $5 \mu \mathrm{g} / \mathrm{ml}$ insulin as previously described. ${ }^{15}$ For serum $(20 \%$ FBS) stimulation experiments, FBS and insulin were omitted from the medium (factor-free medium).

\section{Immunohistochemistry and Immunofluorescence}

Immunohistochemistry staining using specific anticortactin monoclonal antibody 4F11 (Millipore,
Billerica, MA and a kind gift from Dr Weed) were performed on tissue microarrays containing $0.9 \mathrm{~mm}$ cores of 77 nevi, 8 in-situ, 50 invasive and 103 metastatic malignant melanomas constructed as previously described with minor modifications. ${ }^{16}$ Each tissue microarray also contained control normal and tumor tissue from other organs. Individual sections of nevi (6 banal nevi, 10 dysplastic nevi), 1 in-situ, 19 invasive and 3 metastatic melanomas were also used. In addition, a subset of tumors (17 invasive and 2 metastatic) and nevi (14 banal nevi) was also analyzed as cores in TMAs as well as individual sections. The tissue was fixed in $10 \%$ buffered formalin and embedded in paraffin. The use of tissue followed the guideline of an IRBapproved protocol. Deparaffinization, rehydration, antigen retrieval, primary and secondary antibody reaction and detection by diaminobenzidine were all performed on automated Benchmark XT (Ventana Medical Systems, Tucson, AZ) following the manufacture's protocols. The staining was quantitated using Automated Cellular Imaging System (ACISII, Chromavision, San Juan Capistrano, CA). Each slide was scanned at $10 \times$ magnification. Threshold values were adjusted so that the areas with either any appreciable positive staining ('immunopositivity') or very dark staining ('strong immunopositivity') were masked as brown pixels. The blue pixels correlated with areas of counterstain. The percentage value for immunohistochemistry represents the brown area divided by the sum of the brown and blue areas. The intensity of a selected area was the average integrated optical density of the brown in the thresholded brown area, calculated by the system's software. A few cases with significant melanin pigment were discarded from the analysis, and not included in the total numbers of cases stained. To target immunopositivity in relevant lesional cells, on average 3 or 8 representative $400 \times$ fields were selected for a tissue microarray core or an individual section, respectively. Immunopositivity is reported as the multiplication between staining intensity and the percentage of positive staining. ${ }^{17}$ Each case was assigned the highest immunopositivity among the replicate cores. ${ }^{18}$ For immunofluorescent microscopy, the cells plated on glass coverslips were fixed and stained using anti-cortactin (4F11) as previously reported ${ }^{19}$ with minor modifications. Filamentous-actin was detected using rhodaminelabeled phalloidin (Cytoskeleton, Denver, CO).

\section{Fluorescence In-Situ Hybridization (FISH)}

Two P1 genomic cortactin clones ${ }^{20}$ were labeled by nick translation with SpectrumRed conjugated dUTP (Nick-Translation DNA Kit, Abbott Molecular, Abbott Park, IL). Dual-color FISH assays were performed on blank sections of TMAs using a mixture of the SpectrumRed CTTN probe and the 
SpectrumGreen CEP11 commercial probe (Abbott Molecular) according to protocol previously published. ${ }^{21}$ At least 50 nuclei were scored for each core within the selected histological lesion. Based on the percentage of cells with $\leq 2,3$ or $\geq 4$ copies of CTTN, 3 categories of FISH patterns were defined as follows: (a) no genomic gain $(\leq 40 \%$ of cells with $\geq 3$ copies of CTTN); (b) low genomic gain ( $\geq 40 \%$ cells with 3 copies and/or 10 to $<40 \%$ cells with $\geq 4$ copies of CTTN ); (c) high level of genomic gain ( $\geq 40 \%$ of cells with $\geq 4$ copies of CTTN). CTTN gene amplification was defined as either the ratio CTTN/CEP11 $\geq 2$, or presence of clusters $(\geq 4$ signals) of CTTN in $\geq 10 \%$ of scored cells. CTTN gene amplification was categorized as high level of genomic gain.

\section{Statistical Analysis}

The association between CTTN genomic status (no gain, low level or high level gain) detected by FISH and lesion types was tested via Chi-square test. The correlation of the CTTN genomic status with immunohistochemistry (immunopositivity and very strong immunopositivity) scores was calculated by spearman correlation coefficient. Immunohistochemistry scores were compared across lesional types using Wilcoxon test. For patients with invasive tumor, immunohistochemistry scores were compared between metastatic status (yes vs no) via Wilcoxon test. For patients with metastasis, immunohistochemistry scores were compared across tissue types (lung, lymph node or skin metastases) via Wilcoxon test. Paired two-sample t-test was used to compare immunohistochemistry scores in metastases at different times from the same patient. Overall survival and disease-specific survival were analyzed by Cox proportional hazard model for patients with metastasis. An immunohistochemistry score of 63 was found as a significant cut-off in predicting diseasespecific survival in metastases via PARTY Package in $\mathrm{R}$, version 2.6.2. A statistical significant cutoff of 0.05 was used. All analyses were done by SAS software, version 9.1; except otherwise mentioned.

\section{Results}

\section{Cortactin Over-Expression Correlates with Melanocytic Tumor Progression}

Immunohistochemical staining using a specific anticortactin monoclonal antibody was performed on human melanocytic lesions from tissue microarrays and individual sections, including 83 nevi, 10 dysplastic nevi, 9 in-situ, 69 invasive and 106 metastatic melanomas. There was a significant difference in the level of cortactin protein expression in benign vs malignant lesions. Most benign nevi had minimal (Figure 1c) or focal weak immunostaining (Figure 1d). Occasional nevi had focal moderate immunostaining (Figure 1e), and the intensity tended to diminish with maturation upon dermal descent. In contrast, most samples of melanoma had diffuse and intense immunostaining (Figure 2a-f). In a few cases of tumor with paradoxical maturation,,$^{22}$ the smaller cells had less intensity. The quantitative immunopositivity was significantly different between the benign (nevi) and malignant (in-situ, invasive and metastatic melanomas) lesions $(P<0.0001)$. Invasive and metastatic tumors both showed significantly higher level of expression than nevi $(P<0.0001$ for both $)$ (Figure 3a). 74 and $85 \%$ of invasive and metastatic melanomas, respectively, had cortactin expression levels that exceeded the 95th percentile found in nevi. Furthermore, metastatic melanomas had a significantly higher immunopositivity than invasive tumors $(P=0.02)$. Interestingly, within the nevi, the immunopositivity was higher in dysplastic than in banal nevi $(P=0.04)$. The staining intensity was also quantified to identify which lesions contained very strong immunopositivity. As shown in Figure 3b, only melanomas were very strongly positive for cortactin immunostaining, with a highly significant difference compared to nevi $(P<0.0001)$. Within the tumors, metastatic melanomas had higher values than invasive tumors $(P=0.0008)$.

To further demonstrate the increased expression of cortactin in melanoma tissues using a different methodology, equal amount of total protein extracted from sections of nevi and melanomas were analyzed using anti-cortactin immunoblotting. The signal of cortactin protein (seen as intact p80/85, and its major breakdown product) was significantly higher in melanoma samples (data not shown).

The abnormality in cortactin expression was not limited to the intensity, as different distribution patterns were seen in nevi and melanomas. As shown in Figure 1d and e, in nevi, the weak staining was evenly distributed in the cytoplasm. In contrast, in most melanomas the strong staining was accentuated in the cell periphery (Figure 2a, c, d and f), similar to the distribution of cortactin in the membrane ruffles and lamellipodia in cultured melanoma cells (Figure $3 a, b$ and d) and other cultured cell types. ${ }^{19}$ A few tumors also showed an abnormal dot-like distribution (Figure 2e), reminiscent of cortactin localization in podosomes of src-transformed cells. ${ }^{23}$ We hypothesize that the altered subcellular localization may reflect an additional mechanism for protein dysregulation during tumorigenesis, in addition to upregulation of the level of protein expression.

\section{Cortactin Co-Localizes with F-Actin and Displays a Distinctive Distribution in Human Melanoma Cells}

To determine the localization of cortactin in melanoma cells, immunofluorescent staining was performed on cultured human melanoma cell lines. In addition to the pool of protein in the perinuclear 

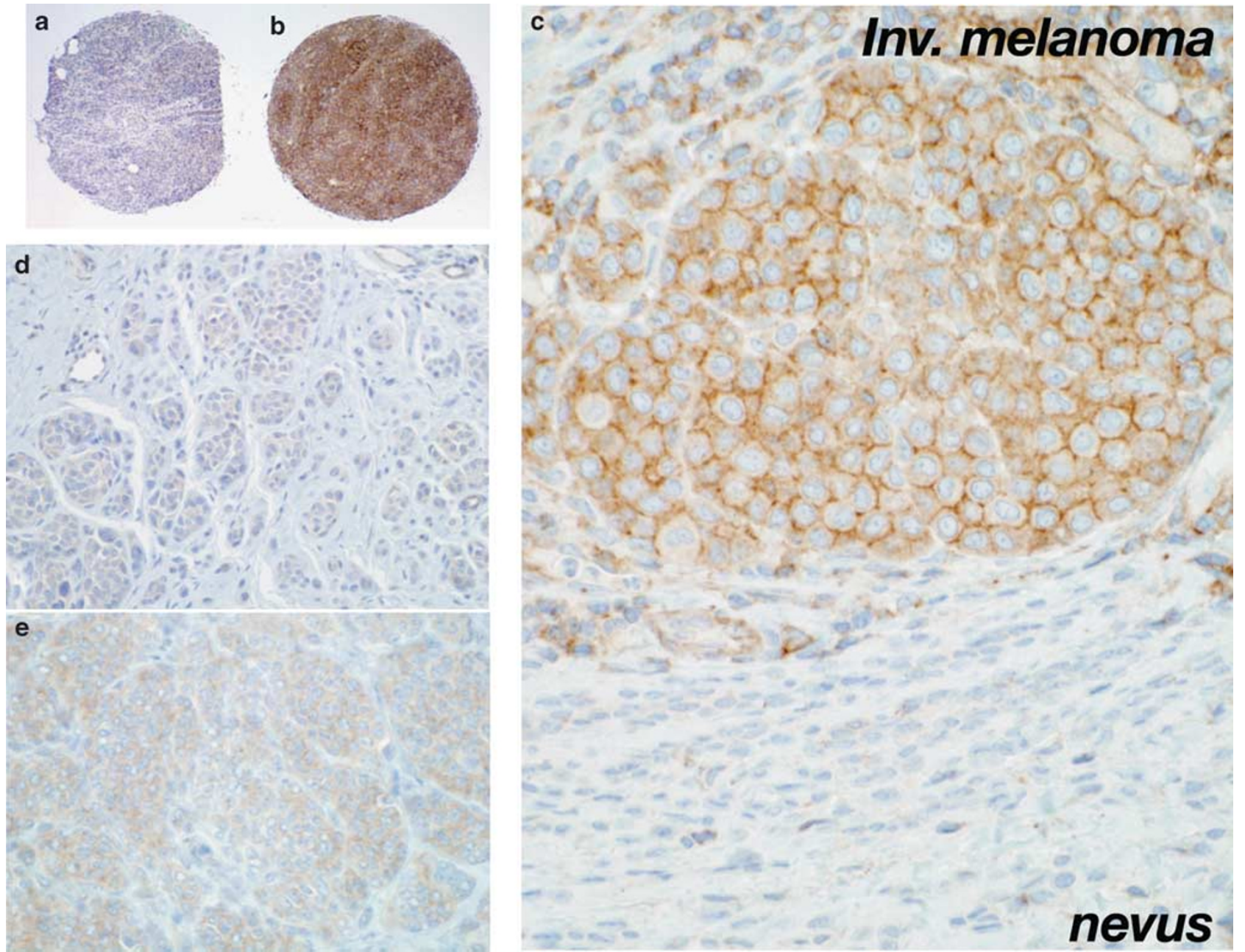

Figure 1 Immunohistochemical staining of cortactin in human melanocytic tumors: example cores in tissue microarrays (a-nevus, b-metastatic melanoma). (c) Strong staining in an invasive melanoma in contrast to minimal staining in a pre-existing nevus. (d and e) Focal weak (d) and focal moderate (e) staining in benign nevi, note the even cytoplasmic distribution.

area without co-localization with F-actin in the cell cortex, presumably in association with the Golgi complex, ${ }^{24}$ there was a prominent cell border distribution. The staining in the cell cortex and leading edge of the cells co-localized with F-actin (Figure 4a and b). Serum-starved melanoma cells showed diminished cell cortex localization (Figure 4c), likely due to inactivation of the small GTPase Rac1. ${ }^{25}$ After serum stimulation, cortactin redistributed to the membrane ruffles, co-localizing with Factin (Figure 4d). Since most of the cellular functions involving cortactin occur in the cell cortex, these findings suggest that the subcellular localization of cortactin may be another important aspect of altered protein regulation in the tumor cells.

\section{Cortactin Gene Amplification is Uncommon in Melanocytic Tumors}

Since CTTN gene amplification is a major mechanism for protein overexpression in breast and head and neck carcinomas, FISH analyses were performed on the TMAs to look for CTTN gene copy numbers in nevi and melanomas. No gene amplification was found in 87 nevi analyzed (Figure 5a). CTTN gene amplification was present in six of 160 melanomas tested (3.75\%): 1 of 46 invasive, 5 of 96 metastatic, and 0 of 18 in-situ tumors. In all six cases, the gene amplification was seen as small clusters of CTTN signal in the nuclei, and the tumors showed corresponding high-level cortactin protein expression (Figure 5e and f). However, overall the correlation between CTTN copy number and immunopositivity was not statistically significant $(P=0.18)$. These results suggest that CTTN amplification does not play a major role in aberrant cortactin protein overexpression in melanoma.

In many tumors with CTTN genomic gain, this gene was not classified as amplified because of concomitant increased signals of CEP11. Nevertheless, this may be a reflection of genomic alteration of the tumors. As shown in Table 1, there was a progressive aneuploidy from in-situ to invasive and 

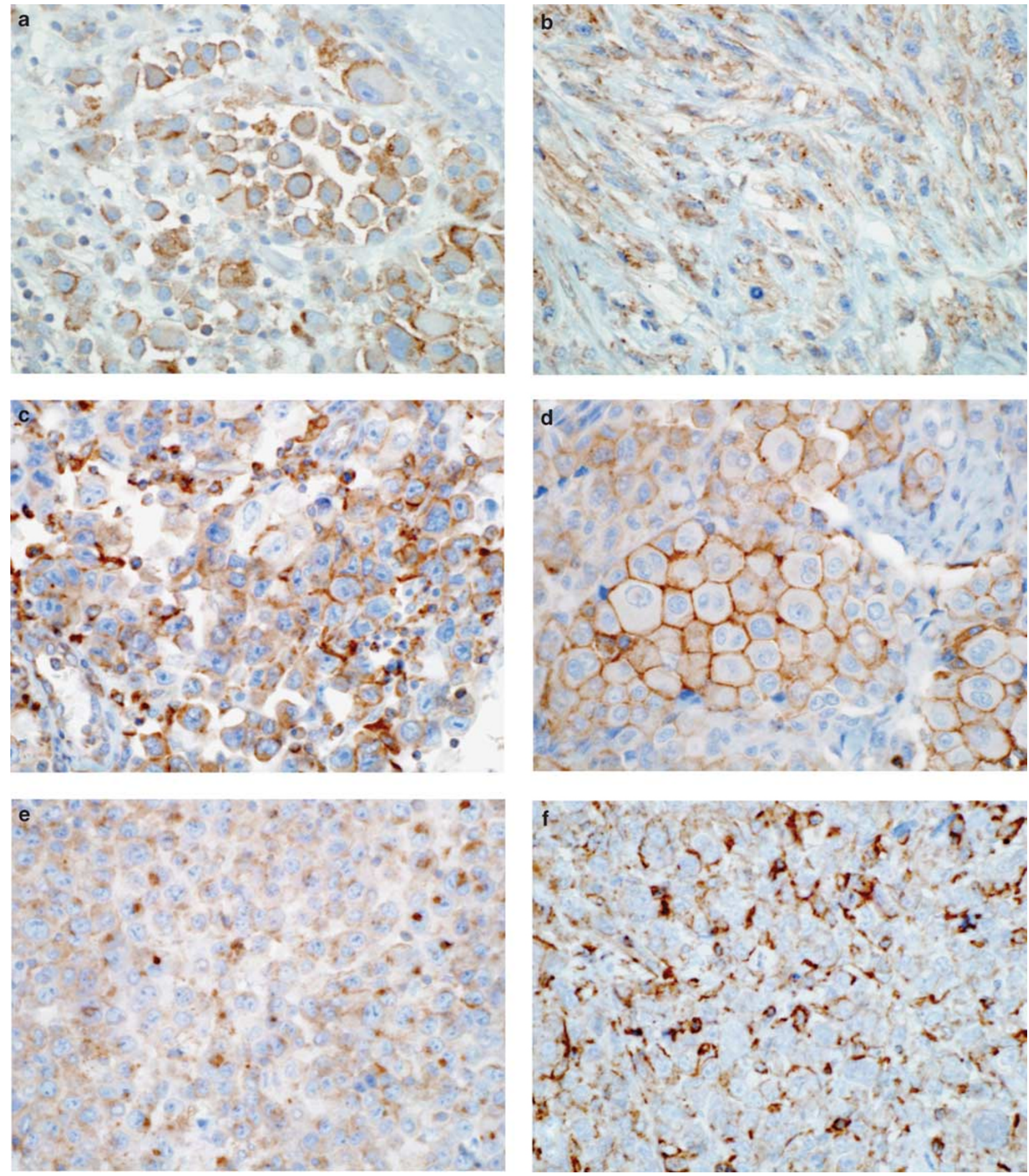

Figure 2 Immunohistochemical staining of cortactin in human melanomas. (a and b) Strong staining in invasive melanoma, with an accentuated cell periphery (a) and dot-like (b) distribution. (c-f) Increased expression in metastatic melanomas. The protein is most commonly seen at the cell periphery (c, $\mathbf{d}$ and $\mathbf{f}$ ), with resemblance to membrane ruffles seen in cultured cells. In occasional cases, it is seen as a globule in the cytoplasm (e).

metastatic melanomas. Nearly half of the invasive and metastatic tumors showed genomic gain, and $20 \%$ of the metastatic tumor had high-level genomic gain. This difference was statistically significant (in-situ vs invasive $P=0.007$, in-situ vs metastatic $P=0.006$ ). The difference between the invasive and metastatic tumors did not reach statistical significance $(P=0.74)$. 



Figure 3 (a and b) Comparison of cortactin expression in nevi, dysplastic nevi (CDN), melanoma in-situ (MIS), invasive and metastatic melanomas. The box indicates 25 th To 75 th percentile of the values; line within the box indicates median; * indicates mean. (c) KaplanMeier survival plot of cortactin expression in metastatic melanomas.

\section{Cortactin Overexpression Correlates with Poor Clinical Outcome in Melanoma Patients}

To determine whether aberrant cortactin expression has clinical significance, we correlated clinical follow-up data with levels of cortactin expression. Eighty-seven patients with metastatic tumors had clinical followup. They were 51 male and 36 female; aged 27-94 years (mean 63, median 64). As shown in Figure 3c, patients with metastatic tumors that had high levels of cortactin expression (immunopositivity score $>63$ ) were more likely to die from their disease (hazard ratio $=3.3, P<0.0001,95 \%$ confidence interval 1.8-6.0).
Several other comparisons showed a trend, however did not achieve statistically significant $P$-values. Invasive melanomas with subsequent metastasis $(n=27)$, compared with the ones without metastasis $(n=42)$, tended to have higher values of immunopositivity and very strong immunopositivity by univariant analysis $(P=0.087$ and 0.156 , respectively). For patients $(n=9)$ who had two metastases at different time points, the immunopositivity and very strong immunopositivity scores tended to be higher in the later metastases $(P=0.22$ and 0.17 , respectively). There was also a trend of higher immunopositivity and very strong immunopositivity values in metastases to the lung, com- 

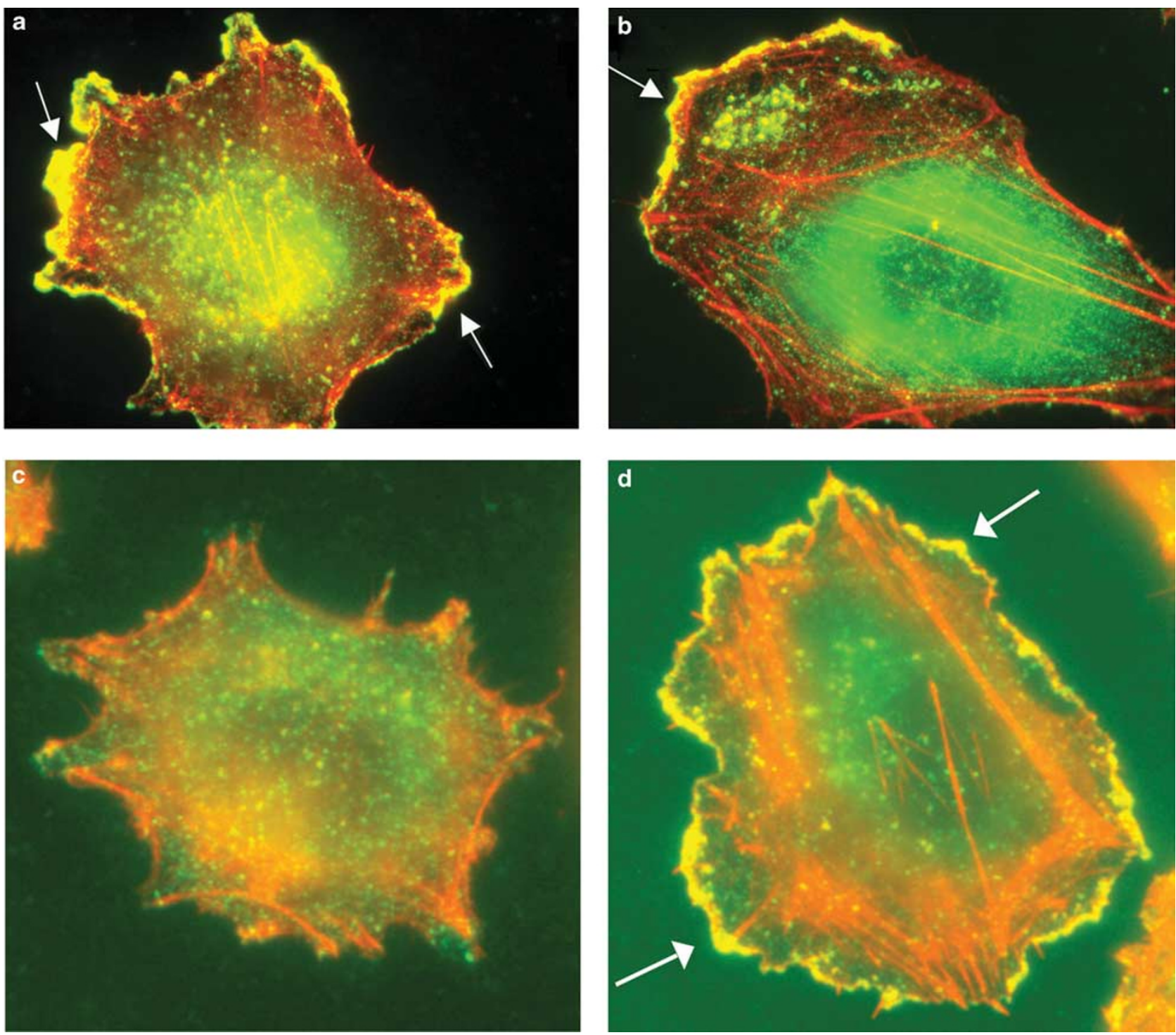

Figure 4 Immunofluorescent staining with anti-cortactin Ab 4F11 (green) and F-actin (phalloidin, red) showed cortactin colocalization with F-actin in the advancing leading edges (yellow, arrows) in melanoma cell lines WM9 (a) and WM11158 (b). The same markers showed re-distribution of cortactin to the membrane ruffles (arrows), colocalizing with F-actin (yellow) in melanoma cell line 1205Lu after serum stimulation (d). Control serum-starved 1205Lu cell (c).

pared with nodal $(P=0.1$ for both $)$ and skin metastases $(P=0.3$ for both).

\section{Discussion}

In this report, we have shown that cortactin protein expression is significantly increased in malignant melanomas. The aberrant distribution pattern seen in the tumor tissues corresponds to the localization of the protein in the cell cortex and leading edges of cultured melanoma cells. Increased cortactin expression correlates with disease progression (metastatic tumors had higher level of expression than invasive tumors). Moreover, high-level cortactin expression in metastatic tumors correlates with poor disease-specific survival. These observations in human tissues support the hypothesis generated from experimental data ${ }^{13,14}$ that cortactin is important in melanoma tumor progression, and provides a rational for potential development of new prognostic biomarkers and targeted therapies.

The importance of cortactin in tumor cell biology is supported by what we know of its basic biochemical and cellular functions. The multidomain structure of cortactin makes it an ideal adaptor as well as a scaffolding protein in regulating the formation and stabilization of nascent branched actin networks, a key process in the remodeling of the cortical actin cytoskeleton that is required for a wide variety of cellular functions. ${ }^{9}$ Cortactin has been shown to be an important component in the 

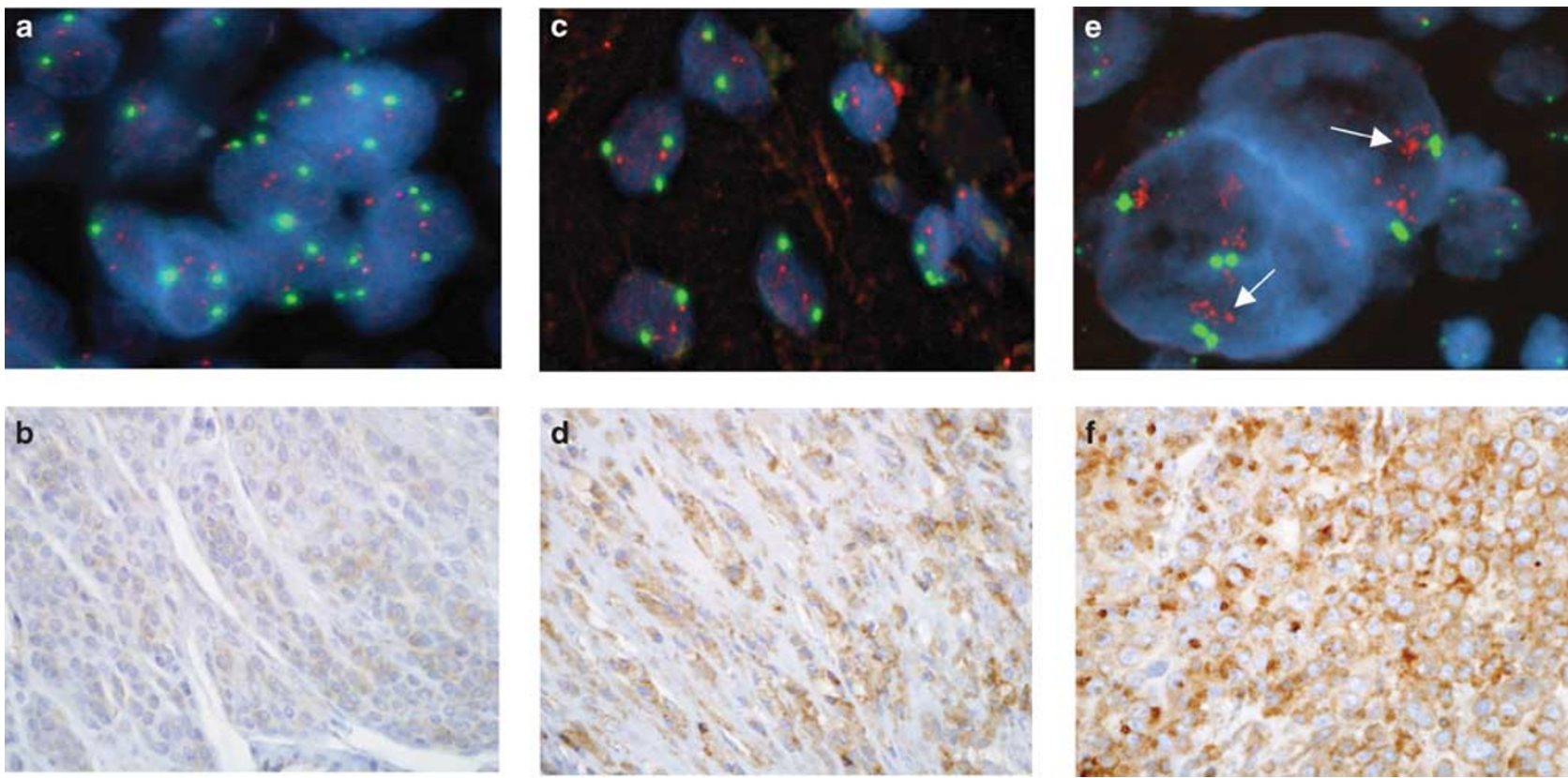

Figure 5 FISH analysis (a, c and $\mathbf{e}$ ) and immunohistochemical staining (b, d, and $\mathbf{f}$ ) of the same samples in a nevus (a and b), and melanomas without (c and $\mathbf{d}$ ) and with (e and f) CTTN amplification. Arrows indicate amplification of CTTN seen as clusters of CTTN probe (red dots); green dots represent probe for centromeric region of chromosome 11.

Table 1 Genomic status of CTTN in melanomas detected by FISH

\begin{tabular}{lcccl} 
Genomic status & $\begin{array}{c}\text { No gain } \\
(\%)\end{array}$ & $\begin{array}{c}\text { Low level } \\
\text { of gain (\%) }\end{array}$ & $\begin{array}{l}\text { High level P-value } \\
\text { of gain (\%) }\end{array}$ \\
\hline $\begin{array}{l}\text { In-situ }(n=18) \\
\text { Invasive }(n=46)\end{array}$ & $15(83)$ & $3(17)$ & $0(0)$ & \\
& $21(46)$ & $19(41)$ & $6(13)$ & $\begin{array}{l}0.007 \\
\text { (vs in-situ) }\end{array}$ \\
$\begin{array}{l}\text { Metastatic } \\
(n=96)\end{array}$ & $53(55)$ & $24(25)$ & $19(20)$ & $\begin{array}{l}0.006 \\
\text { (vs in-situ) }\end{array}$ \\
\hline
\end{tabular}

FISH, fluorescent in-situ hybridization.

subcellular structures involved in cell motility (lamellapodia, filapodia and membrane ruffles), extracellular matrix anchorage, degradation and cell invasion (osteoclast podosomes, tumor cell podosomes and invadopodia) and receptor trafficking (endocytic vesicles and receptor stimulated dorsal 'waves') (reviewed in refs. 5,6,26). Aberrant cortactin expression in melanoma cells may lead to increased sensitivity to extracellular chemotactic signals. Cortactin showed translocation to the cell cortex upon serum stimulation. Serum and growth factors known to regulate melanocyte proliferation (bFGF, PDGF, pleiotrophin) ${ }^{16,27}$ increased phosphorylation of cortactin on Src targeted residue Y421. In melanoma cell line $1205 \mathrm{Lu}$, cortactin was associated with src protein kinase and $\mathrm{N}$-cadherin $(\mathrm{Xu} \& \mathrm{Wu}$, unpublished data). The aberrant cortactin overexpression may result in increased sensitivity of melanocytic cells to the stimulation of growth factors in the epidermal and dermal environment, thus potentially contributing to the exit from cell cycle arrest, and to phenotypic transformation of the tumor cells by decreasing their dependence on extracellular growth and chemotactic molecules. ${ }^{28}$ Increased cortactin expression may also contribute to melanoma cell phenotypic transformation that is analogous to epithelium-mesenchymal transition. In normal adult human tissue, cortactin is highly expressed in tissue and cell types with actin-based contractile ability, such as smooth muscle cells and myoepithelial cells. ${ }^{29}$ Nevic cells are for the most part, static cells whose actin cytoskeleton is likely to be rigid and stable with a purpose of providing structural support. We propose that acquisition of increased levels of cortactin, a protein normally involved in active cell shape remodeling, cell migration and rapid response to environmental stimuli, will contribute to a more motile and invasive cellular phenotype. CTTN gene amplification was not a common event in melanomas, similar to adenoid cystic carcinoma of the salivary gland, but different from breast and head and neck carcinomas where protein overexpression is commonly associated with gene amplification..$^{711,30}$ The mechanism(s) for protein overexpression in melanomas remains to be determined, may be due to higher mRNA level or increased protein stability. ${ }^{30}$

Components of the actin cytoskeleton have been proposed as targets for cancer drug development. ${ }^{31}$ Animal models to study tumor metastasis using breast, squamous or hepatocellular carcinoma cells have demonstrated that overexpression of cortactin lead to increased in vivo metastases (reviewed in 
ref. 7). In a breast cancer cell line, a cell-permeable peptide as well as a small-molecule compound that can inhibit cortactin binding to a partner protein AMAP1 effectively inhibits tumor cell invasion in vitro and lung metastasis in vivo. This peptide can also inhibit in vitro invasion of glioblastoma and lung tumor cells lines. ${ }^{32}$ These studies, taken together with our results, indicate the potential of cortactin as an interesting biomarker for melanoma prognostication and a target for therapeutic development.

\section{Acknowledgements}

The work was supported by a Research Career Development Award from the Dermatology Foundation to $\mathrm{H} \mathrm{Wu}$ and by $\mathrm{NIH}$ grants R01DE014578 and P20 RR16440 to S Weed. The authors thank Dr Jonathan Chernoff for his generous support to the laboratory work and input to the manuscript, Dr Peter Adams for his helpful comments to the manuscript. The authors thank Robert Page and Karen Trush for their excellent technical support.

\section{Disclosure/conflict of interest}

The authors declare no conflict of interest.

\section{References}

1 Clark WH, Elder DE, Guerry DI, et al. A study of tumor progression: the precursor lesions of superficial spreading and nodular melanoma. Hum Pathol 1984;15:1147-1165.

2 Miller AJ, Mihm MC. Mechanisms of disease: melanoma. N Engl J Med 2006;355:51-65.

3 Yamaguchi H, Condeelis J. Regulation of the actin cytoskeleton in cancer cell migration and invasion. Biochim Biophys Acta 2007;1773:642-652.

4 Pawlak G, Helfman DM. Cytoskeletal changes in cell transformation and tumorigenesis. Curr Opin Genet Dev 2001;11:41-47.

5 Weed SA, Parsons JT. Cortactin: coupling membrane dynamics to cortical actin assembly. Oncogene 2001;20:6418-6434.

6 Daly RJ. Cortactin signalling and dynamic actin networks. Biochem J 2004;382:13-25.

7 Weaver AM. Cortactin in tumor invasiveness. Cancer Letters 2008;265:157-166.

8 Ammer AG, Weed SA. Cortactin branches out: roles in regulating protrusive actin dynamics. Cell Motil Cytoskeleton 2008;65:687-707.

9 Goley ED, Welch MD. The ARP2/3 complex: an actin nucleator comes of age. Nat Rev Mol Cell Biol 2006;7:713-726.

10 Rodrigo JP, Garcia LA, Ramos S, et al. EMS1 gene amplification correlates with poor prognosis in squamous cell carcinomas of the head and neck. Clin Cancer Res 2000;6:3177-3182.
11 Schuuring E. The involvement of the chromosome 11q13 region in human malignancies: cyclin D1 and EMS1 are two new candidate oncogenes-a review. Gene 1995;159:83-96.

12 Hui R, Ball JR, Macmillan RD, et al. EMS1 gene expression in primary breast cancer: relationship to cyclin D1 and oestrogen receptor expression and patient survival. Oncogene 1998;17:1053-1059.

13 Ayala I, Baldassarre M, Giacchetti G, et al. Multiple regulatory inputs converge on cortactin to control invadopodia biogenesis and extracellular matrix degradation. J Cell Sci 2008;121:369-378.

14 Huang J, Asawa T, Takato T, et al. Cooperative roles of Fyn and cortactin in cell migration of metastatic murine melanoma. J Biol Chem 2003;278: 48367-48376.

15 Satyamoorthy K, Li G, Vaidya B, et al. Insulin-like growth factor-I-induced migration of melanoma cells is mediated by interleukin-8 induction. Cell Growth Diff 2002;13:87-93.

$16 \mathrm{Wu} \mathrm{H}$, Barusevicius A, Babb J, et al. Pleiotrophin expression correlates with melanocytic tumor progression and metastatic potential. J Cut Pathol 2005;32: 125-130.

17 Hes O, Michal M, Kuroda N, et al. Vimentin reactivity in renal oncocytoma: immunohistochemical study of 234 cases. Arch Pathol Lab Med 2007;131:1782-1788.

$18 \mathrm{Kim} \mathrm{M}$, Gans JD, Nogueira C, et al. Comparative oncogenomics identifies NEDD9 as a melanoma metastasis gene. Cell 2006;125:1269-1281.

$19 \mathrm{Wu} \mathrm{H}$, Parsons JT. Cortactin, an 80/85-kilodalton pp60src substrate, is a filamentous actin-binding protein enriched in the cell cortex. J Cell Biol 1993;120:1417-1426.

20 Yuan BZ, Zhou X, Zimonjic DB, et al. Amplification and overexpression of the EMS 1 oncogene, a possible prognostic marker, in human hepatocellular carcinoma. J Mol Diagn 2003;5:48-53.

21 Cappuzzo F, Toschi L, Domenichini I, et al. HER3 genomic gain and sensitivity to gefitinib in advanced non-small-cell lung cancer patients. Br J Cancer 2005;93:1334-1340.

22 Ruhoy SM, Prieto VG, Eliason SL, et al. Malignant melanoma with paradoxical maturation. Am J Surg Pathol 2000;24:1600-1614.

$23 \mathrm{Wu} \mathrm{H}$, Reynolds AB, Kanner SB, et al. Identification and characterization of a novel cytoskeletonassociated pp60src substrate. Mol Cell Biol 1991;11: 5113-5124.

24 Cao H, Orth JD, Chen J, et al. Cortactin is a component of clathrin-coated pits and participates in receptormediated endocytosis. Mol Cell Biol 2003;23: 2162-2170.

25 Weed SA, Du Y, Parsons JT. Translocation of cortactin to the cell periphery is mediated by the small GTPase Rac1. J Cell Sci 1998;111(Pt 16):2433-2443.

26 Cosen-Binker LI, Kapus A. Cortactin: the grey eminence of the cytoskeleton. Physiology 2006;21: 352-361.

27 Shih IM, Herlyn M. Autocrine and paracrine roles for growth factors in melanoma. In Vivo 1994;8:113-123.

28 Timpson P, Wilson AS, Lehrbach GM, et al. Aberrant expression of cortactin in head and neck squamous cell carcinoma cells is associated with enhanced cell proliferation and resistance to the epidermal growth factor receptor inhibitor gefitinib. Cancer Res 2007;67: 9304-9314. 
$29 \mathrm{Wu} \mathrm{H}$, Montone KT. Cortactin localization in actincontaining adult and fetal tissues. J Histochem Cytochem 1998;46:1189-1191.

30 Greer Jr RO, Said S, Shroyer KR, et al. Overexpression of cyclin D1 and cortactin is primarily independent of gene amplification in salivary gland adenoid cystic carcinoma. Oral Oncol 2007;43: 735-741.
31 Rao J, Li N. Microfilament actin remodeling as a potential target for cancer drug development. Curr Cancer Drug Targets 2004;4:345-354.

32 Hashimoto S, Hirose M, Hashimoto A, et al. Targeting AMAP1 and cortactin binding bearing an atypical src homology 3/proline interface for prevention of breast cancer invasion and metastasis. Proc Natl Acad Sci USA 2006;103:7036-7041. 